\title{
Hands up: a case of bilateral inferior shoulder dislocation
}

\author{
K S Kumar, S O’Rourke, J G Pillay
}

\begin{abstract}
Inferior shoulder dislocation (luxatio erecta) is a rare form of shoulder dislocation. A case involving bilateral luxatio erecta with bilateral brachial plexus injury and rotator cuff tears is reported. A brief review of presentation and management of this condition is presented. (Emerg Med f 2001;18:404-405)
\end{abstract}

Keywords: shoulder dislocation

Inferior dislocation of the gleno-humeral joint, or luxatio erecta, is a rare form of shoulder dislocation. We report a case of bilateral inferior dislocation with bilateral brachial plexus injury and rotator cuff tears.

\section{Case report}

A 58 year old butcher was brought to our accident and emergency department having slipped and fallen in the garden while under the influence of alcohol. The patient remained undiscovered for two hours as he lay on the ground with both hands held fixed above his head. On arrival the patient was not hypothermic but in considerable distress. Physical examination revealed that both shoulder joints were held in $100^{\circ}$ abduction and external rotation with immediate pain on attempted passive movement. Full neurological assessment was difficult but wrist drop and diffuse sensory deficit was present bilaterally.

The patient was prescribed intravenous morphine for pain control. Radiographs confirmed the clinical diagnosis of bilateral inferior dislocation (fig 1). He was transferred to the resuscitation room and reduction was achieved without difficulty by axial traction/counter traction under sedation and collar and cuffs were applied. Repeat radiographs confirmed reduction and there were no accompanying fractures of the greater tuberosity, inferior glenoid or acromion. On neurological reassessment there was paraesthesia from $\mathrm{C} 5$ to $\mathrm{T} 1$ in both upper limbs. His motor power (MRC power grading) on the right side was 2 in 30 October 2000
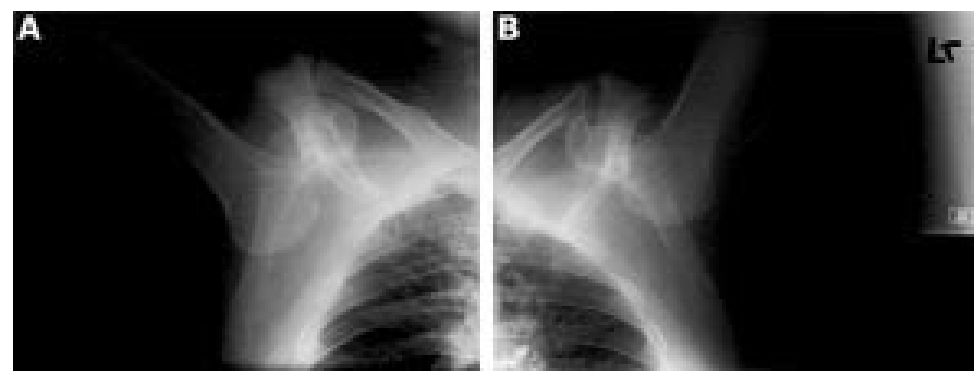

Figure 1 Radiographs showing right $(A)$ and left (B) inferior dislocation of shoulder. shoulder abduction, 3 in elbow extension and flexion and 2 in wrist extension and flexion. On the left side it was 2 in shoulder abduction, 4 in elbow extension and flexion, 2 in wrist extension and 4 in wrist flexion. This was consistent with diffuse brachial plexus injury, more pronounced on the right side. Dynamic wrist splints were applied and the patient was admitted overnight.

Two weeks later physiotherapy was started with passive movement of the upper limbs. At five weeks the motor weakness on the left side was much improved but no better on the right with the sensory deficit as before. There was evidence of bilateral rotator cuff injury. A decision was made to arrange for electromyography and nerve conduction studies, which confirmed the bilateral brachial plexus injury with evidence of regeneration. Magnetic resonance imaging showed bilateral partial rotator cuff tears and a normal cervical spine. Six months after the initial injury the patient has returned to work but with persistent weakness of wrist extension, elbow flexion and extension and shoulder abduction, especially on the right side.

\section{Discussion}

Inferior dislocation of the shoulder (luxatio erecta) is rare accounting for an estimated $0.5 \%$ of shoulder dislocations. ${ }^{1}$ First described by Middeldorpf and Scharm in 1859, ${ }^{2}$ it occurs when the shoulder is forced into hyperabduction with the proximal humerus being levered over the acromion process. ${ }^{3}$ It may also result from direct axial loading on the fully abducted arm. On physical examination the affected side is held above the head with the shoulder abducted and externally rotated. Any attempt to passively adduct the shoulder will cause severe pain. It is estimated that $60 \%$ of these patients have some neurological deficit of the upper limb prior to reduction, usually deltoid "badge patch" numbness, but any deficit usually resolves post-reduction. ${ }^{4}$ It is essential to look for clinical evidence of brachial plexus injury in patients with inferior dislocation of shoulder as it is this which causes the resultant morbidity rather than the dislocation itself. Radiological investigation will show the shaft of the humerus lying parallel to the spine of the scapula and the articular surface of the humeral head directed inferiorly without contact with the glenoid. Reduction is achieved by applying traction in line with the humerus. A sheet placed inferior to the joint is pulled upwards by an assistant as countertraction. The arm is then gently adducted.

Bilateral inferior dislocation of the glenohumeral joint is extremely rare. Murrad 
described the first case in $1920^{5}$ and only four further cases have been described since. ${ }^{6}$ What makes this patient unusual is the resultant bilateral rotator cuff tears, with bilateral brachial plexus injury.

Mr Pillay is the guarantor of the paper.

1 Laskin RS, Sedlin ED. Luxatio erecta in infancy. Clin Orthop 1971;71:126-9.
2 Middeldorpf $M$, Scharm B. De nova humeri luxationis speie. Clinique Europenne 1859;2:12-16.

3 Newman KJH, Bendall R. Bilateral inferior shoulder dislocation: both subglenoid and subcoracoid types seen in the same patient. Injury 1993;24:684-5.

4 Mallon WJ, Basset PH, Goldner RD. Luxatio erecta; the inferior glenohumeral dislocation. F Orthop Trauma 1990;4: 19.

5 Murard J. Un cas de luxatio erecta de 1'epaule, double et symetrique. Rev Orthop 1920;7:423-9.

6 Gelczer RK, Swee RG, Adkins MC. Bilateral inferior glenohumeral dislocations. F Trauma 1996;40:825-6. 